\title{
Development of MC-based uncertainty estimation technique of unfolded neutron spectrum by multiple-foil activation method
}

\author{
Katsumi Aoki ${ }^{1, *}$, Tadahiro Kin ${ }^{1}$, Md Kawchar Ahmed Patwary $^{1}$, Naoto Araki ${ }^{1}$, Kosuke Yoshinami $^{1}$, Masaya Yamaguchi $^{1}$, \\ Masatoshi Itoh $^{2}$, and Yukinobu Watanabe ${ }^{1}$ \\ ${ }^{1}$ Department of Advanced Energy Engineering Science, Kyushu University, 6-1 Kasuga-koen, Kasuga, Fukuoka, 816-8580, Japan \\ ${ }^{2}$ Cyclotron and Radioisotope Center (CYRIC), Tohoku University, 6-3 Aoba, Aramaki, Aoba-ku, Sendai, Miyagi, 980-8578, Japan
}

\begin{abstract}
Unfolding process plays an important role in neutron energy distribution measurement by means of the multiple-foil activation method. We have developed an algorithm for estimating uncertainty of resultant neutron distribution. For the demonstration of the algorithm, we have conducted an experiment to measure thick-target neutron yield (TTNY) of the $\mathrm{C}(d, n)$ reaction induced by $20-\mathrm{MeV}$ deuterons with the multiple-foil activation method. The experiment was conducted at Cyclotron and Radioisotope Center, Tohoku University in Japan. The obtained experimental data were analyzed by the algorithm. As a result, we found that uncertainty of derived TTNY has dependency on neutron emission energy. In addition, the dependency is caused by excitation functions of activation reactions. Therefore, we found the particular reactions causing large uncertainty in TTNY. The result of present study shows that the algorithm can evaluate uncertainty and its causes.
\end{abstract}

\section{Introduction}

Recently, the accelerator-based neutron source has been applied in various fields such as boron neutron capture therapy (BNCT) [1], radioisotopes (RIs) production [2, 3], nuclear transmutation [4], and nuclear fusion engineering [5]. A high intensity neutron source is necessary in these applications.

We have been focusing on the RI production study using accelerator-based neutron generated by the $\mathrm{C}(d, n)$ reaction. To obtain intense neutron, a thick neutron converter made of carbon is used. The generated neutrons irradiate raw material, and then an $\mathrm{RI}$ is produced inside the raw material. Because neutron energy distribution of the reaction can be adjusted by incident deuteron energy, radioactivity and isotopic purity of the RI can be controlled. This method is a part of "generation of radioisotopes with accelerator neutrons by deuteron (GRAND)" proposed by Nagai et al. [2]. For instance, production of ${ }^{64} \mathrm{Cu}$ [6], ${ }^{92} \mathrm{Y}$ [7], and ${ }^{99 m} \mathrm{Tc}$ [8] were previously proposed in the GRAND project.

For clinical use, estimation of radioactivity and purity of the RI is important. In the estimation, simulation study is a powerful method, because in some cases, stable isotopes are produced as byproducts which can not be directly measured by a radiation detector. Moreover, the simulation study is necessary for design of an efficient irradiation system and radiation shielding calculation. For precise simulation, accurate thick-target neutron yields (TTNYs) as functions of neutron energy and neutron emission angle are required. In the RI production method, the TTNYs

*e-mail: aoki.katsumi.473@s.kyushu-u.ac.jp from 10 to $50 \mathrm{MeV}$ are required, but the data has not been systematically measured.

The multiple-foil activation method is effective for measurement of TTNY. In the method, TTNY should be derived by using unfolding method. For the unfolding method, numbers of produced atoms $N_{i}$ and production rate functions $R_{i j}$ of interested reactions are necessary to derive TTNY $\phi_{j}$, where $i$ and $j$ express reaction ID and the neutron energy group, respectively. The following equation expresses the relation of these values.

$$
N_{i}=\sum_{j} R_{i j} \phi_{j}
$$

Since derivation of $\phi_{j}$ is an inverse problem that cannot be solved analytically, the unfolding method is necessary. In the past, various unfolding algorithms have been developed, for example, an iterative approximation method, a maximum entropy method, and an iterative Bayesian method. Each algorithm has unique characteristics [9]. GRAVEL [10] or SAND-II code [11], whose algorithm are the iterative approximation method, is widely used for the unfolding process. They also require initial guess spectrum and convergence conditions. However, both codes can not give uncertainty in the resultant neutron energy distribution.

To overcome the situation, we have developed an algorithm for estimating uncertainty of TTNY caused by the unfolding process. 


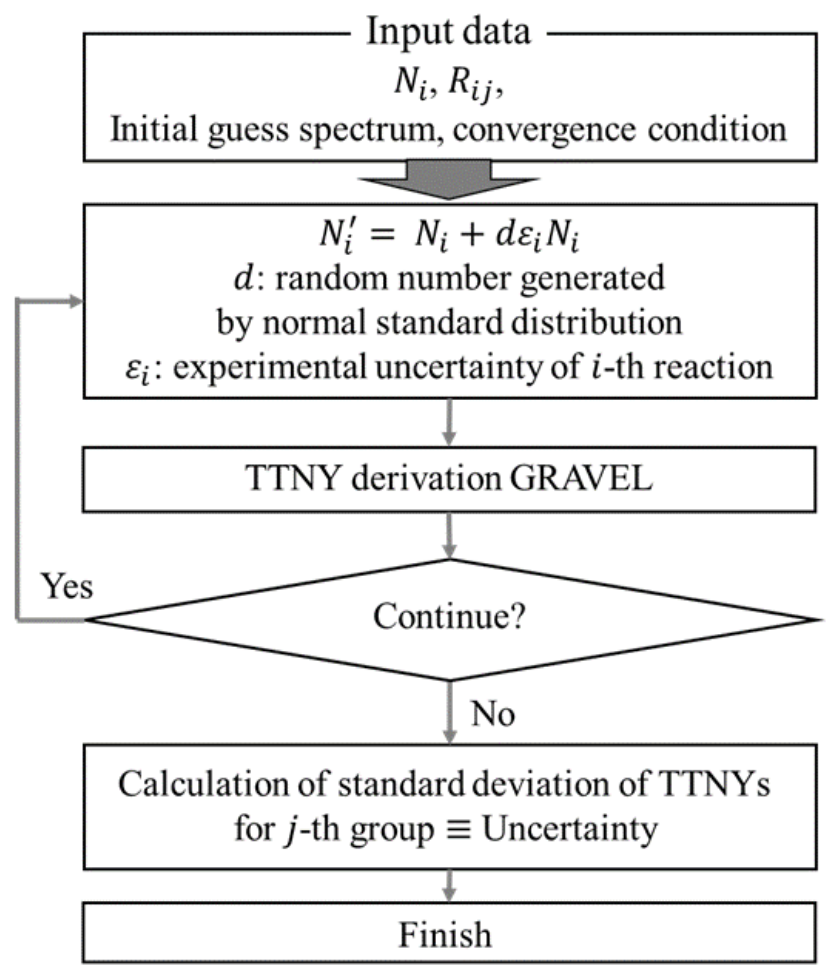

Figure 1. Flow chart of Monte-Carlo based uncertainty estimation algorithm. $N_{i}, R_{i j}, N_{i}^{\prime}, d$, and $\varepsilon_{i}$ show number of atoms of produced atoms in experiment, production rate function, value generated by using normal random number, normal random number, and experimental uncertainty, respectively.

\section{Development of uncertainty estimation algorithm}

We proposed a Monte-Carlo based uncertainty estimation algorithm for GRAVEL code. The flow chart of the algorithm is shown in Fig. 1.

The algorithm gives a large number of distorted TTNYs as shown in Fig. 2 (a) caused by experimental uncertainty. First, $N_{i}^{\prime}$ is calculated by following equation to take experimental uncertainty into account:

$$
N_{i}^{\prime}=N_{i}+d \varepsilon_{i} N_{i}
$$

where $d$ and $\varepsilon_{i}$ represent a random number generated by standard normal distribution and experimental relative uncertainty of $i$-th reaction. Next, we can obtain propagated uncertainty at each energy group as the standard deviation of the TTNYs as shown in Fig. 2 (b).

\section{Experiment}

To demonstrate the algorithm, we conducted an experiment to measure TTNY of the $\mathrm{C}(d, n)$ reaction by means of multiple-foil activation method at Cyclotron and Radioisotope Center (CYRIC), Tohoku University in Japan. Deuterons were accelerated to $20 \mathrm{MeV}$ by an AVF930 cyclotron and bombarded on a thick carbon target ( $23 \mathrm{~mm} \times$ $23 \mathrm{~mm} \times 2 \mathrm{~mm}^{\mathrm{t}}$ ) to produce accelerator-based neutrons. The neutrons irradiated on square-shaped multiple foils $(50 \mathrm{~mm} \times 50 \mathrm{~mm})$ made of ${ }^{27} \mathrm{Al}\left(0.025 \mathrm{~mm}^{\mathrm{t}}\right),{ }^{\text {nat }} \mathrm{Fe}(0.02$
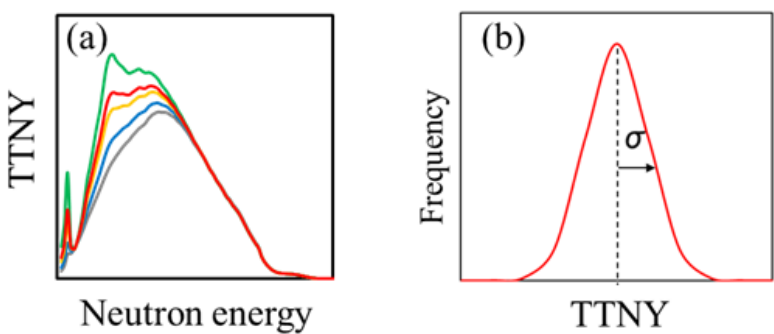

Figure 2. (a) The distribution of distorted TTNYs, and (b) deviation of TTNYs in an energy range.

$\left.\mathrm{mm}^{\mathrm{t}}\right),{ }^{59} \mathrm{Co}\left(0.1 \mathrm{~mm}^{\mathrm{t}}\right),{ }^{\text {nat }} \mathrm{Ni}\left(0.1 \mathrm{~mm}^{\mathrm{t}}\right),{ }^{\text {nat }} \mathrm{Zn}\left(0.2 \mathrm{~mm}^{\mathrm{t}}\right)$, ${ }^{\text {nat }} \mathrm{Zr}\left(0.1 \mathrm{~mm}^{\mathrm{t}}\right)$, and ${ }^{\text {nat }} \mathrm{Mo}\left(0.05 \mathrm{~mm}^{\mathrm{t}}\right)$. The foils were placed at $1215 \mathrm{~mm}$ downstream to the neutron converter at 0 degrees to the deuteron beam. Average deuteron beam current was $2.0 \mu \mathrm{A}$ during 19-hours irradiation. Figure 3 shows the schematic view of the irradiation system. After that, gamma rays emitted from the irradiated foils were measured with a HP Ge detector.

\section{Results and Discussion}

First, the number of produced atoms of interested reactions were obtained from gamma-ray spectra analysis as shown in Table 1 .

Second, to calculate production rate functions, the cross sections stored in JENDL-4.0 [12] were multiplied 


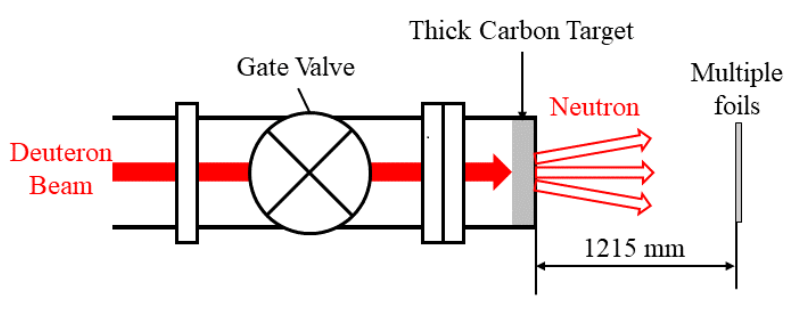

Figure 3. Schematic view of the irradiation setup.

Table 1. Interested reactions, the number of produced atoms of the reactions, and statistical uncertainty.

\begin{tabular}{ccc}
\hline $\begin{array}{c}\text { Activation } \\
\text { reaction }\end{array}$ & $\begin{array}{c}\text { Number of } \\
\text { produced atoms }\end{array}$ & $\begin{array}{c}\text { Statistical } \\
\text { uncertainty }\end{array}$ \\
\hline${ }^{27} \mathrm{Al}(n, \alpha){ }^{24} \mathrm{Na}$ & $5.30 \times 10^{7}$ & $0.43 \%$ \\
$\left.{ }^{56} \mathrm{Fe}(n, p)\right)^{56} \mathrm{Mn}$ & $1.28 \times 10^{7}$ & $1.07 \%$ \\
${ }^{58} \mathrm{Ni}(n, 2 n){ }^{57} \mathrm{Ni}$ & $1.24 \times 10^{7}$ & $0.83 \%$ \\
${ }^{59} \mathrm{Co}(n, \alpha){ }^{56} \mathrm{Mn}$ & $1.90 \times 10^{7}$ & $0.59 \%$ \\
$\left.{ }^{59} \mathrm{Co}(n, p)\right)^{59} \mathrm{Fe}$ & $2.68 \times 10^{8}$ & $0.52 \%$ \\
${ }^{64} \mathrm{Zn}(n, p){ }^{64} \mathrm{Cu}$ & $6.91 \times 10^{8}$ & $1.38 \%$ \\
${ }^{67} \mathrm{Zn}(n, p){ }^{67} \mathrm{Cu}$ & $7.59 \times 10^{6}$ & $1.02 \%$ \\
${ }^{96} \mathrm{Zr}(n, 2 n){ }^{95} \mathrm{Zr}$ & $7.00 \times 10^{7}$ & $1.08 \%$ \\
$\left.{ }^{96} \mathrm{Mo}(n, p)\right)^{96} \mathrm{Nb}$ & $1.63 \times 10^{6}$ & $1.52 \%$ \\
\hline
\end{tabular}

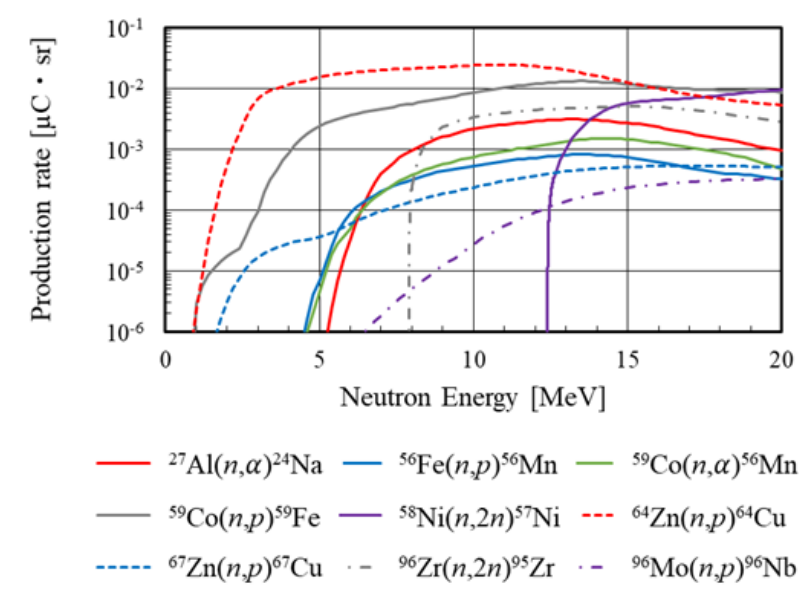

Figure 4. Production rate functions of interested reactions.

by solid angle of each foil, total charge of deuteron beam and surface density of atoms. The calculated production rate functions are shown in Fig. 4.

Third, initial guess spectrum was calculated by DEURACS [13] which is a theoretical calculation code for deuteron induced reactions.

We derived the TTNY and its uncertainty by using the present algorithm. Figure 5 shows two-dimensional histogram of 1,000 TTNYs. To calculate the propagated uncertainty, we made projection of TTNYs in each neutron energy group. As an example of the projection, histogram between 6.3 and $6.5 \mathrm{MeV}$ is shown in Fig. 6. The standard deviation of the histogram represents propagated uncertainty in the unfolding process. We calculated the standard deviation for every neutron energy group.

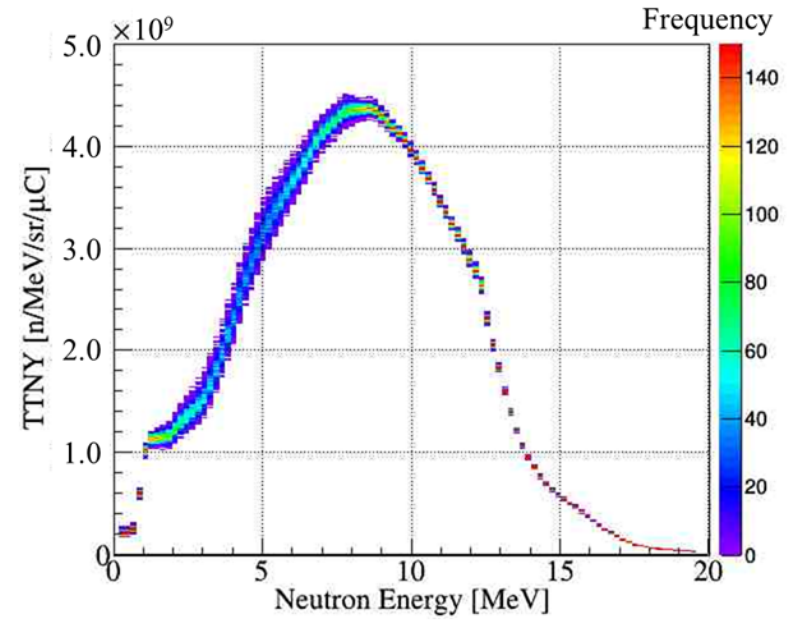

Figure 5. The two-dimensional histogram of 1,000 TTNYs.

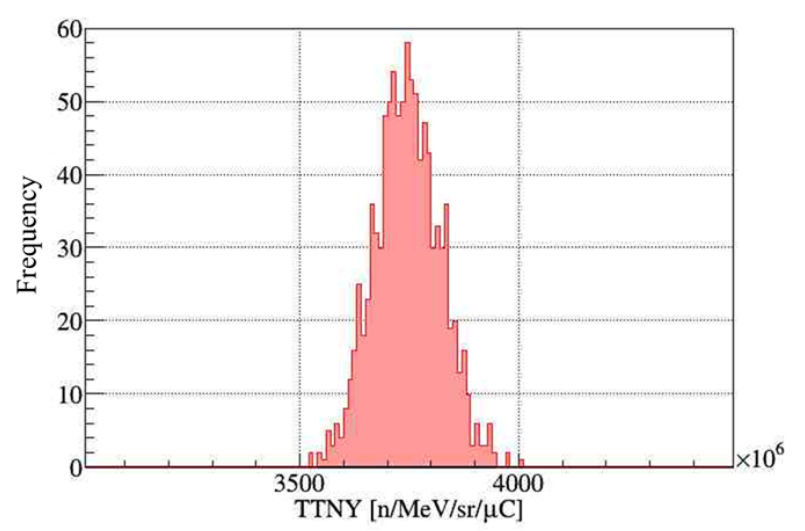

Figure 6. The histogram made projection of TTNYs between 6.3 and $6.5 \mathrm{MeV}$.

Obtained three-sigma uncertainty is shown as error bar in Fig. 7. We found that the uncertainty has neutron energy dependency. To investigate the dependency, we derived TTNY deviation for each reaction independently. In the calculation, we fixed number of produced atoms except that of single reaction in the present algorithm. The parameter $\varepsilon_{i}$ is set to $10 \%$ for observation of sensitive energy range of the single reaction. The sensitive neutron energy range can be observed as deviation of derived TTNYs.

These results are shown in Fig. 8. From these results, large relative standard deviation is observed below $7 \mathrm{MeV}$ only for three reactions having lower threshold energies, ${ }^{59} \mathrm{Co}(n, p){ }^{59} \mathrm{Fe},{ }^{64} \mathrm{Zn}(n, p){ }^{64} \mathrm{Cu}$, and ${ }^{67} \mathrm{Zn}(n, p){ }^{67} \mathrm{Cu}$. Therefore, large uncertainty in low energy range shown in Fig. 7 is led by the three reactions.

\section{Summary and conclusion}

We have developed an algorithm for estimating uncertainty of TTNY derived by unfolding method. The MonteCarlo based algorithm can apply to any unfolding codes. For demonstration, we applied it for an accelerator-based neutron measurement by means of multiple-foil activation method using GRAVEL code in unfolding process in the 


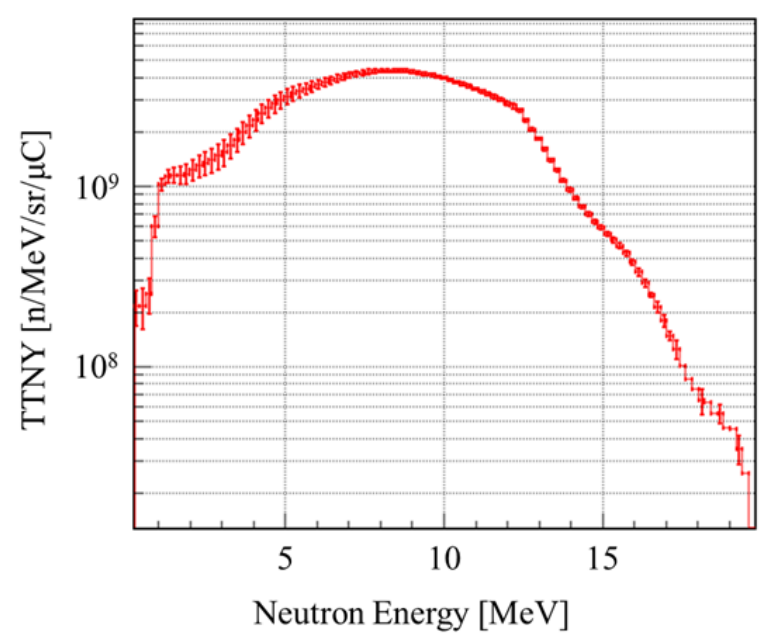

Figure 7. Obtained TTNY and its three-sigma uncertainty by using the present algorithm.

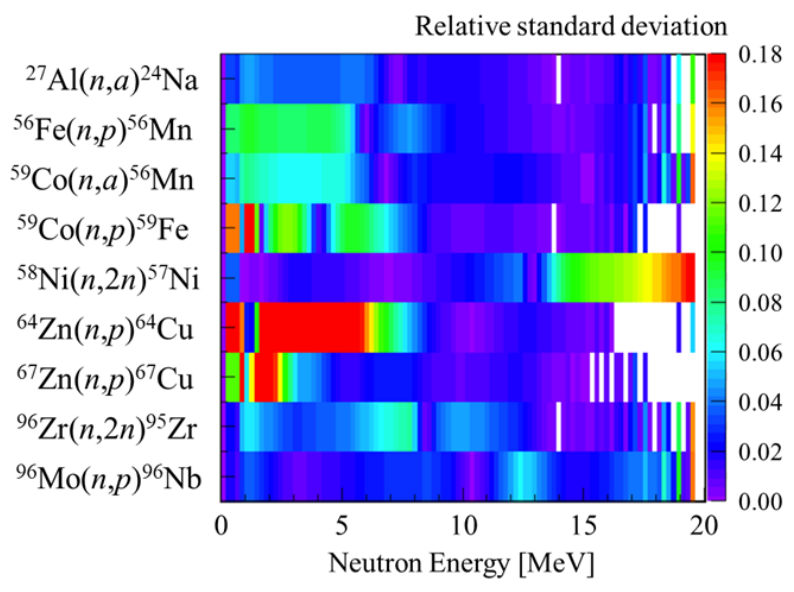

Figure 8. Obtained relative standard deviation for each reaction by using the algorithm.

present study. We derived the TTNY and its uncertainty by using the present algorithm. As a result, the uncertainty has neutron energy dependency. Single reaction contribution was surveyed to investigate the energy dependency. From the survey, we observed sensitive neutron energy range for each dosimetry reaction. We found that large deviation appears below $7 \mathrm{MeV}$ only for three reactions, ${ }^{59} \mathrm{Co}(n, p){ }^{59} \mathrm{Fe},{ }^{64} \mathrm{Zn}(n, p){ }^{64} \mathrm{Cu}$, and ${ }^{67} \mathrm{Zn}(n, p){ }^{67} \mathrm{Cu}$.
In the future, we will apply the algorithm to other unfolding codes based on the maximum entropy, iterative Bayesian and artificial neural network methods.

\section{References}

[1] M. Yoshioka, Review of Accelerator-based Boron Neutron Capture Therapy Machines, in Proceedings, 7th International Particle Accelerator Conference (IPAC 2016): Busan, Korea, May 8-13, 2016 (2016), p. THXB01

[2] Y. Nagai, K. Hashimoto, Y. Hatsukawa, H. Saeki, S. Motoishi, N. Sato, M. Kawabata, H. Harada, T. Kin, K. Tsukada et al., Journal of the Physical Society of Japan 82, 064201 (2013)

[3] T. Kin, M.K.A. Patwary, M. Kamida, K. Aoki, N. Araki, K. Yoshinami, Y. Watanabe, M. Itoh, Journal of the Physical Society of Japan 24, 011031 (2019)

[4] Implications of Partitioning and Transmutation in Radioactive Waste Management, Number 435 in Technical Reports Series (2004), ISBN 92-0115104-7

[5] A. Moeslang, V. Heinzel, H. Matsui, M. Sugimoto, Fusion Engineering and Design 81, 863 (2006), proceedings of the Seventh International Symposium on Fusion Nuclear Technology

[6] T. Kin, T. Kawagoe, S. Araki, Y. Watanabe, Journal of Nuclear Science and Technology 54, 1123 (2017)

[7] T. Kin, Y. Sanzen, M. Kamida, Y. Watanabe, M. Itoh, EPJ Web Conf. 146, 08009 (2017)

[8] Y. Nagai, Y. Hatsukawa, Journal of the Physical Society of Japan 78, 033201 (2009)

[9] K. Aoki, T. Kin, M. Kamida, N. Araki, Y. Watanabe, M. Itoh, Journal of the Physical Society of Japan 24, 011033 (2019)

[10] M. Matzke, Tech. Rep. PTB-N-19, Braunschweig Univ. Phys.-Tech. Bundesanst, Braunschweig (1994)

[11] W. N. McElroy, S. Berg, T. Crockett, R. G. Hawkins, A STUDY OF THE ITERATIVE METHOD 1, 125 (1967)

[12] K. Shibata, O. Iwamoto, T. Nakagawa, N. Iwamoto, A. Ichihara, S. Kunieda, S. Chiba, K. Furutaka, N. Otuka, T. Ohsawa et al., Journal of Nuclear Science and Technology 48, 1 (2011)

[13] S. Nakayama, H. Kouno, Y. Watanabe, O. Iwamoto, K. Ogata, Phys. Rev. C 94, 014618 (2016) 